\title{
Dynamical Evolution of Globular Cluster Systems
}

\author{
E. Vesperini \\ Department of Physics and Astronomy, Michigan State University, East \\ Lansing, $M I, U S A$
}

\begin{abstract}
I present the results of a number of simulations of the dynamical evolution of globular cluster systems (GCS) in elliptical galaxies including the effects of two-body relaxation, dynamical friction, stellar evolution and the effects of the tidal field of the host galaxy. The results of detailed models for the evolution of the M87 GCS are also presented. A significant number of clusters are disrupted by evolutionary processes and the properties of many of the clusters which survive are effected by dynamical evolution. In spite of large differences in the efficiency of evolutionary processes in different galaxies, the final galaxy-to-galaxy variation of the GCS mean mass and its radial variation within individual galaxies in my simulations are small and consistent with observations. The effects of dissolution of low-concentration clusters due to mass loss through stellar evolution are also discussed and are shown to play an important role in the evolution of a power-law GCS mass function (GCMF), similar to that observed in young cluster systems in merging galaxies, towards a final GCMF with properties consistent with observations.
\end{abstract}

\section{Introduction}

A number of theoretical studies have shown that, due to the effects of two-body relaxation, tidal shocks, stellar evolution, the structure and the stellar content of globular clusters evolve and that, during the evolution, clusters lose mass and eventually dissolve (see e.g Meylan \& Heggie 1997 for a review). Although many of the effects predicted by theoretical investigations of the dynamical evolution of globular clusters have been confirmed by observational studies, there are a number of observational findings concerning the global properties of GCS which are apparently in conflict with what one would expect if dynamical evolution played an important role in shaping the current GCS properties. In particular, observations show that the overall shape of the GCMF and the mean mass of clusters in galaxies with different structure are very similar to each other and that, within individual galaxies, the mean cluster mass does not significantly depend on the galacto-centric radius. Since the efficiency of evolutionary processes depends on the structure of the host galaxy and, within individual galaxies, on the distance from the center of the host galaxy, these observational findings have sometimes been interpreted as an indication that evolutionary processes did not play an important role in determining the properties of GCS. In order to explore this issue I have a carried out a large number of simulations to study the dynamical evolution of GCS in elliptical galaxies with different properties. I summarize below the main results concerning the GCMF evolution; further details can be found in Vesperini (2000), Vesperini (2001), Vesperini \& Zepf (2003) and Vesperini et al. (2003). 


\section{Results}

1) For a log-normal initial GCMF similar to that observed in old GCS, the final values of the mean mass and the galaxy-to-galaxy variation of the mean mass, from simulations of the evolution of GCS in elliptical galaxies with properties equal to those observed, are perfectly consistent with observations. In most galaxies dynamical evolution is important and evolutionary processes lead to the disruption of a significant fraction of the initial population of clusters. Although the efficiency of evolutionary processes and the fraction of surviving cluster depend on the properties of the host galaxy, the galaxy-to-galaxy variation of the final mean mass is small and consistent with observations. The radial variation of the final mean mass within individual galaxies is small and in agreement with observational data.

2) In simulations with a power-law initial GCMF, evolutionary processes transform the power-law GCMF into a bell-shaped GCMF similar to that of old GCS, but the final mean cluster masses are for most galaxies smaller than those observed, and the galaxy-to-galaxy-variation and the radial variation of the mean mass are large and not consistent with observations.

3) The results of detailed models for the evolution of the M87 GCS show that, for a power-law initial GCMF, the final values of the GCS mean mass and its radial variation are consistent with observations if a strongly anisotropic initial GCS velocity distribution is adopted, but the final kinematical properties of these models are characterized by a strong radial anisotropy inconsistent with observational kinematical data. A number of models starting with a bell-shaped (in $\log M$ ) initial GCMF similar to that of old GCS have final properties satisfying all the observational constraints available on the GCMF, the kinematics and the spatial distribution of the M87 GCS.

4) A number of simulations including a distribution of initial concentrations for individual clusters, and including the dissolution of low-concentration clusters induced by mass loss due to stellar evolution have been carried out. The results of these simulations show that dissolution of low-concentration clusters can significantly affect the initial number of clusters in a GCS, alter the initial GCMF, and play an important role in the evolution of a power-law initial GCMF towards a final GCMF with properties consistent with observations.

\section{References}

Meylan, G., \& Heggie, D.C., 1997, A\&A Rev., 8, 1

Vesperini, E. 2000, MNRAS, 318, 841

Vesperini, E. 2001, MNRAS, 322, 247

Vesperini, E., \& Zepf, S. E., 2003, ApJ, 587, L97

Vesperini, E., Zepf, S. E., Kundu, A., \& Ashman, K. M., 2003, ApJ, 593, 760 